\title{
A study of the outcome of pregnancy complicated by obstetric cholestasis
}

\author{
Samik Medda*, Sibani Sengupta, Upasana Palo
}

Department of Obstetrics and Gynecology, Vivekananda Institute of Medical Sciences, Kolkata, West Bengal, India

Received: 28 December 2017

Revised: 21 January 2018

Accepted: 24 January 2018

\author{
*Correspondence: \\ Dr. Samik Medda, \\ E-mail: samikmedda@gmail.com
}

Copyright: () the author(s), publisher and licensee Medip Academy. This is an open-access article distributed under the terms of the Creative Commons Attribution Non-Commercial License, which permits unrestricted non-commercial use, distribution, and reproduction in any medium, provided the original work is properly cited.

\begin{abstract}
Background: Obstetric cholestasis is one of the most common causes of liver disease in pregnancy. Present study was carried out to study the incidence of Obstetric Cholestasis and its feto-maternal outcome in a tertiary care hospital.

Methods: It is a prospective epidemiologycal study during a period of one year (2014 to 2015) over 100 pregnant ladies suffering from pruritus and detected as having Obstetric Cholestasis. They were followed up and maternal as well as fetal-neonatal outcome recorded. Appropriate statistical analysis done as applicable.

Results: The incidence of Obstetric Cholestasis in our hospital was 9.9\%. Majority of cases (43.0\%) are diagnosed in late gestational age, mostly during 28 to 32 weeks period of gestation. Maternal morbidities are due to sleep disturbance (60/100), dyslipidemia, coagulation abnormality, PPH (10.0\%) and increase chance of operative delivery $(66.0 \%)$. Neonatal morbidities are mainly due to fetal distress, prematurity (22.0\%), low birth weight (32/100) and meconium staining of amniotic fluid (42.0\%). Maximum number of patients are delivered at 37 to 38 weeks, due to active and early intervention.

Conclusions: Early diagnosis and active maternal and fetal surveillance is of utmost importance to avoid adverse outcomes.
\end{abstract}

Keywords: Epidemiological study, Obstetric cholestasis, Outcome

\section{INTRODUCTION}

Obstetric cholestasis is one of the most common causes of liver disease in pregnancy. It is associated with significantly high adverse maternal and fetal outcome. ${ }^{1}$ Obstetric cholestasis (OC) or Intrahepatic cholestasis of pregnancy (ICP) is a cholestatic disorder characterized by unexplained pruritus during pregnancy with elevated serum bile acids $(>10 \mu \mathrm{mol} / \mathrm{L})$ and/or transaminases in late second and third trimester of pregnancy, in absence of other liver disease, and spontaneous resolution of signs and symptoms within two to three weeks after delivery. ${ }^{1,2}$

Obstetric cholestasis has been observed in almost all ethnic groups, but there is relevant geographical variation in its incidence. ${ }^{1}$ The incidence of $\mathrm{OC}$ among Indian women has been reported to be about $1 \%$.,

Obstetric cholestasis classically manifests in second or third trimester with pruritus and deranged liver function test. The etiology of ICP is mostly unknown, but it is believed to be multifactorial with genetic, environmental or hormonal factors being involved. ${ }^{5-7}$ Impairment of the function of major hepatocellular canalicular transporters lead to cholestasis.

Obstetric cholestasis is associated with significant maternal morbidities. The main maternal impact for women with cholestasis is pruritus with no skin changes, worse at night and most intense in the palms of the hands 
and soles. ${ }^{5-7}$ They have an increased risk for postpartum hemorrhage, dyslipidemia, preterm labour and operative interference. $^{7,8}$

Intrahepatic Cholestasis of pregnancy can have devastating consequences for the fetus with perinatal mortality reaching up to $11 \%$ to $20 \%$ in untreated cases ${ }^{6}$. Adverse fetal outcomes associated with the conditions include preterm labour, preterm prelabour rupture of membrane, fetal distress, abnormal CTG, meconium staining, spontaneous intrauterine death. ${ }^{5,9-11}$

Ursodeoxycholic acid (UDCA) is considered to be a safe treatment option in the later part of pregnancy. ${ }^{12,13}$ The condition typically resolves within 48 hours of women giving birth, with biochemical markers predominantly becoming normal within 2-4 weeks postnatally. ${ }^{5}$

Present study is aimed to detect the incidence of OC in our hospital and follow up those pregnancies to evaluate maternal and perinatal outcome.

\section{METHODS}

Our prospective epidemiological study was performed in Ramakrishna Mission Seva Prathishthan Hospital, Kolkata over one year (May 2014 to April 2015). The diagnosis of obstetric cholestasis was made by clinical symptom of pruritus without a skin rash affecting mainly extremities and worsening at night, associated with biochemical evidence of cholestasis in form of elevated serum transaminases (ALT and AST) with or without elevated serum bilirubin, in the absence of other liver disease. Postpartum resolution was studied in reference to improvement of pruritus and abnormal Liver Function Tests after 6 weeks of delivery.

History taking, clinical examination and laboratory investigations were carried out to diagnose obstetric cholestasis and calculate disease frequency.

Among them, initial 100 cases were included in the study as sample cases for monitoring clinical nature of the disease, relevant biochemical alterations and outcome of pregnancy. Other relevant investigations were done to exclude other conditions of altered LFT like Hepatitis serology, hepato-billiary ultrasonography, liver autoimmune screen, etc.

LFT was repeated every 2-4 weeks interval as required. Fasting lipid profiles and coagulation profiles were also detected. In present study serum levels that are more than upper limit of pregnancy specific reference ranges are considering as positive for OC. However, due some limitation, measurement of serum bile acid could not be done. All patients included in the study were given ursodeoxycholic acid 300-1200 mg/day in divided doses for the rest of the antenatal period.
Maternal outcome was studied in reference to insomnia due to severe pruritus, associated dyslipidemia and deranged coagulation profile (increase PT), mode of delivery, preterm labour, preterm pre-labour rupture of membrane, postpartum hemorrhage.

Fetal outcome was studied in reference to prematurity, abnormal CTG, fetal distress or hypoxia, meconium stained liquor, low birth weight (less than $2.5 \mathrm{~kg}$ ) or Intra Uterine Growth Restriction, NICU admission rate and perinatal death (IUFD/Still born).

\section{Statistical analysis}

Statistical Analysis was performed with the help of Epi Info (TM) 3.5.3 as per the recommendations of the Centers for Disease Control and Prevention (CDC). Test of proportion (Z-test) and t-test were used to test the significant difference. A $p$ value $\leq 0.05$ was considered statistically significant.

\section{RESULTS}

The total number of deliveries during the one year study period, were 3876 in this hospital, among them 384 patients had Obstetric Cholestasis. So, the incidence of OC was $9.9 \%$. The incidence of ICP were $9.7 \%$ in primigravida (167 of 1719) and $10.0 \%$ in multigravida (217 of 2157) accordingly showing no significant difference $(\mathrm{Z}=0.07 ; \mathrm{p}=0.94)$ (Table 1$)$.

Table 1: Incidence of $\mathrm{OC}$ according to gravida.

\begin{tabular}{|llll|}
\hline Parity & $\begin{array}{l}\text { Total } \\
\text { number }\end{array}$ & $\begin{array}{l}\text { Obstetric } \\
\text { cholestasis }\end{array}$ & $\%$ \\
\hline Primigravida & 1719 & 167 & 9.7 \\
\hline Multigravida & 2157 & 217 & 10.0 \\
\hline Total & 3876 & 384 & 9.9 \\
\hline
\end{tabular}

$64.7 \%$ of multigravida women (11 of 17 who had history of previous viable pregnancy) had past history of OC which was significantly higher $(\mathrm{Z}=4.15 ; \mathrm{p}=0.0001)$ (Table 2).

Table 2: Distribution of past history of OC among multipara patients.

\begin{tabular}{|lll|}
\hline Past history & Number & $\%$ \\
\hline Yes & 11 & 64.7 \\
\hline No & 6 & 35.3 \\
\hline Total & 17 & 100.0 \\
\hline
\end{tabular}

The mean age (mean \pm s.d.) of the patients was $27.53 \pm 4.49$ years with range 18-44 years and the median age was 28.0 years. Most of the patients $(44.0 \%)$ were with age between $26-30$ years $(Z=2.51 ; p=0.0121)$. Only $3.0 \%$ of the patients were $>35$ years of age and $6 \%$ were with age $\leq 20$ years (Figure 1 ). 


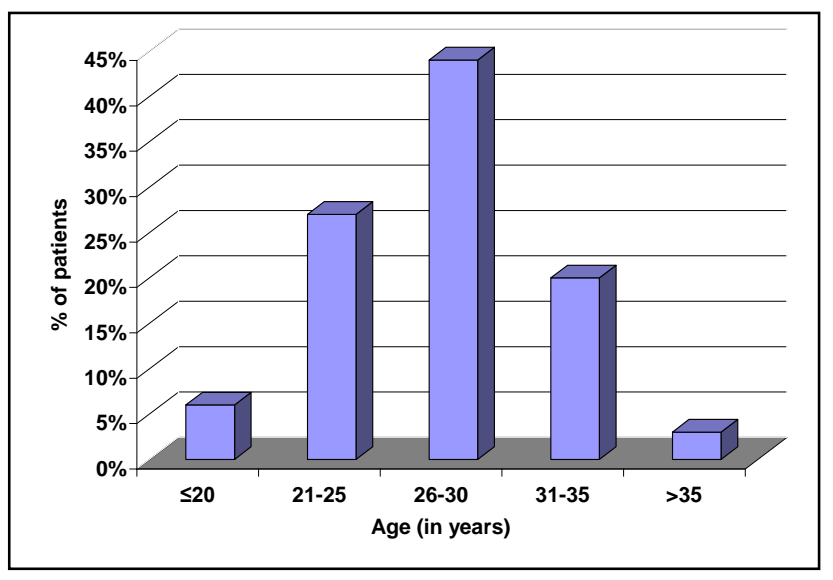

Figure 1: Age distribution of OC in study population.

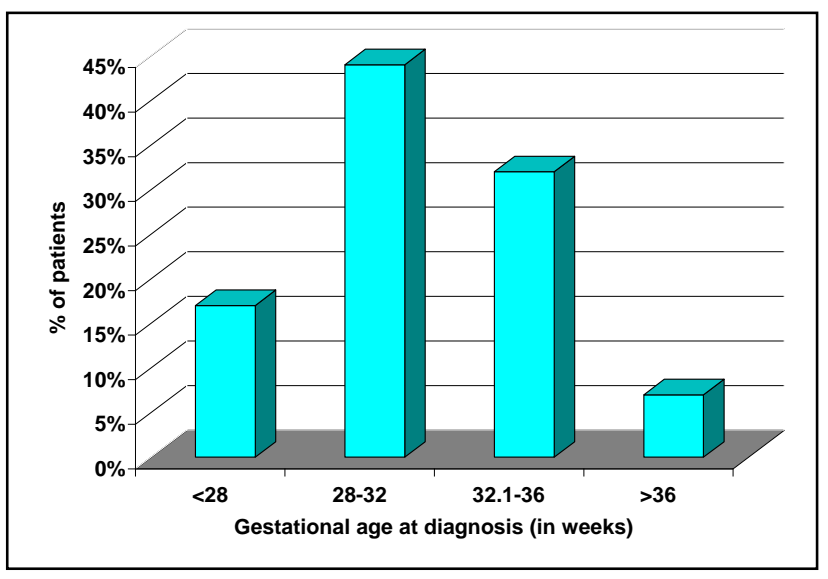

Figure 2: Gestational age at diagnosis (in weeks).

The mean gestational age at diagnosis of the patients was $31.80 \pm 4.39$ weeks with range 17-38 weeks and the median was 32.0 weeks. Most of the patients $(43.0 \%)$ were diagnosed at gestational age of 28-32 weeks followed by $32.1-36$ weeks $(36.0 \%)$ which was significantly higher than that of other gestational age groups $(\mathrm{p}<0.01) .11 \%$ and $10 \%$ patients were diagnosed at $<28$ weeks and $>36$ weeks of gestation respectively (Figure 2).

Table 3: Distribution of maternal outcomes.

\begin{tabular}{|lll|}
\hline Maternal outcome & Number & $\%$ \\
\hline Sleep disturbance & 60 & 60.0 \\
\hline Dyslipidemia & 30 & 30.0 \\
\hline Deranged coagulation profile & 19 & 19.0 \\
\hline PPH & 10 & 10.0 \\
\hline PROM & 10 & 10.0 \\
\hline Operative delivery & 66 & 66.0 \\
\hline Preterm labour spontaneous & 7 & 7.0 \\
\hline
\end{tabular}

Out of total cases of maternal outcomes operative delivery (66\%) and sleep disturbance (60\%) were significantly higher $(\mathrm{p}<0.01)$. It also shows, $30 \%$ patients of OC had dyslipidemia, $19 \%$ had abnormal coagulation profile. Incidence of PPH and PROM were same, $10 \%$ in present study. Also $7 \%$ of patients had spontaneous onset of labour pain before 37 weeks and delivered preterm babies (Table 3 ).

Table 4: Gestational age at delivery (in weeks).

\begin{tabular}{|lll|}
$\begin{array}{l}\text { Gestational age of delivery } \\
\text { (in weeks) }\end{array}$ & Number & $\%$ \\
\hline$<35$ & 2 & 2.0 \\
\hline $35-36$ & 22 & 22.0 \\
\hline $37-38$ & 62 & 62.0 \\
\hline $39-40$ & 14 & 14.0 \\
\hline Total & 100 & 100.0 \\
\hline
\end{tabular}

The mean gestational age of delivery was $37.28 \pm 1.18$ weeks with range $33-40$ weeks and the median was 37.0 weeks. $62 \%$ of the patients delivered between 37-38 weeks which was significantly higher $(\mathrm{p}<0.01)$. Only $2.0 \%$ patients delivered before 35 weeks of gestation and none after 40 completed weeks (Table 4 ).

Table 5: Mode of delivery.

\begin{tabular}{|lll|}
\hline Mode of Delivery & Number & $\%$ \\
\hline Elective CS & 32 & 32.0 \\
\hline Emergency CS & 30 & 30.0 \\
\hline Forceps & 4 & 4.0 \\
\hline Vaginal delivery & 34 & 34.0 \\
\hline Total & 100 & 100.0 \\
\hline
\end{tabular}

Number of the total cases of operative delivery (66\%), which comprised of Elective CS (32\%), Emergency CS $(30 \%)$ and Forceps delivery (4\%), were significantly higher than that of VD $(34 \%)(\mathrm{p}<0.01)$ (Table 5).

Table 6: Distribution of fetal outcomes.

\begin{tabular}{|lll|}
\hline Fetal Outcome & Number & $\%$ \\
\hline Fetal distress & 23 & 23.0 \\
\hline Abnormal CTG & 17 & 17.0 \\
\hline Meconium stained liquor & 41 & 41.0 \\
\hline LBW & 32 & 32.0 \\
\hline IUFD or still born & 2 & 2.0 \\
\hline NICU admission & 27 & 27.0 \\
\hline Preterm birth & 22 & 22.0 \\
\hline
\end{tabular}

Among 100 cases included in present study, 23 patients had evidence of fetal distress $(23.0 \%), 17$ patients had abnormal CTG (17.0\%), 41 patients had meconium stained liquor $(41.0 \%), 22$ patients had preterm birth $(22.0 \%)$ excluding IUFD, 32 patients had delivered low birth weight babies (32.0\%) among them majority had IUGR and others being preterm, 27 neonates required admission to NICU (27.0\%). There were 2 intrauterine fetal deaths $(2.0 \%)$, both delivered preterm. No perinatal death occurred among these cases while 39 patients had no perinatal complications $(39.0 \%)$. Out of total cases of fetal outcome, meconium stained liquor (41\%) and LBW 
$(32 \%)$ were significantly higher $(\mathrm{p}<0.01)$ in present study (Table 6).

Table 7: Post partum resolution after 6 weeks of delivery.

\begin{tabular}{|lll|}
\hline Post partum resolution & Number & $\%$ \\
\hline Normal LFT & 98 & 98.0 \\
\hline Persisting Raised LFT & 2 & 2.0 \\
\hline Total & 100 & 100.0 \\
\hline
\end{tabular}

In present study, in most of the cases normal LFT (98\%) was found $(\mathrm{p}<0.01)$ after 6 weeks of delivery and symptomatically pruritus relieved. So that disease resolved within 6 weeks of delivery (Table 7).

\section{DISCUSSION}

Obstetric Cholestasis is a relatively common cause of hepatic impairment in pregnancy. It has a complex etiology with genetic, endocrine and environmental components. Intrahepatic cholestasis of pregnancy was originally described by Ahlfeld as recurrent jaundice in pregnancy that resolved following delivery. ${ }^{14}$

The incidence of OC shows large variation between different countries and populations. ${ }^{1}$ According to Abedin et al, in the United Kingdom, OC affects only $0.6 \%$ of pregnancies in white Caucasians, but $1.4 \%$ of pregnancies of Indian and Pakistani origin. ${ }^{15}$ The highest incidence of OC $(14 \%)$ has been reported from Chile (Reyes), but a much lower incidence (2-4\%) was found in the latest report (Germain et al). ${ }^{16,17}$ In this study, we found the incidence of ICP was $9.9 \%$, which is comparable to high incidence of $9.3 \%$ reported by Gupta A et al and $8.2 \%$ by Padmaja $\mathrm{M}$ et al. ${ }^{18,19}$ However it is prudent to mention that, our hospital is a tertiary referral center and the incidence of high risk pregnancy is higher. Hence the incidence of $\mathrm{OC}$ is expected to be higher than in community. There was no significant difference in incidence according to parity (primigravida 9.7\% and multigravida $10.0 \%$ ).

Heinonen $S$ et al reported that women of relatively advanced age (>35 years) were at increasing risk of developing OC, but in this study, mean age of the patients was $27.53 \pm 4.49$ years (range 18-44 years) and most of the patients $(44.0 \%)$ were with age between 26-30 years. ${ }^{20}$ This is similar to the results shown by Rasheed $\mathrm{S}$ et al (28 years+ 5.19) and Sosa SY et al $(29.2 \pm 6.8 \mathrm{yrs}) .^{21,22}$ Recurrence of OC in subsequent pregnancies were upto $60-70 \%$ (Ray A et al). ${ }^{23}$ We also found a significantly higher $(64.7 \%)(Z=4.15 ; p=0.0001)$ recurrence rate in multiparous women.

The mean gestational age at diagnosis of ICP was $31.80 \pm 4.39$ weeks and the median were 32.0 weeks. Most of the patients were diagnosed between 28-32 weeks $(43.0 \%)$ and $32.1-36$ weeks $(36.0 \%)$ which was corroborating to the findings of other authors like Dang A et al (mean age $31 \pm 4$ weeks) and Kenyon AP et al (33.7 weeks). ${ }^{3,24}$

This study shows $60 \%$ of the patients had sleep disturbance, due to severe pruritus at night. Generalized pruritus more affecting to palm and sole were the cardinal features without skin rash and clinical jaundice. Total serum bilirubin rarely exceeds $4-5 \mathrm{mg} / \mathrm{dl}$. Serum aminotransferases (ALT and AST) are also elevated 2-10 fold above normal ranges in our patients but none exceed 1000 U/L. ${ }^{1,9}$ Serum levels of alkaline phosphatase may rise up to 7-10 times normal but are difficult to interpret due to elevation of the heat stable placental isoenzyme. Serum total bile acid levels usually exceed $\geq 10 \mu \mathrm{mol} / \mathrm{L}$.

In this study, we treated our patients with topical emollients like calamine lotion and oral Ursodeoxycholic Acid (300-1200) in divided doses. We found complete symptomatic improvement in $65 \%$ cases and partial response in $30 \%$. Rest $5 \%$ of the patients did not get relieved of pruritus. Biochemical improvement, evidenced by decreasing Transaminases levels, was observed in $85 \%$ cases $(\mathrm{p}<0.01)$. Deveer $\mathrm{R}$ et al found serum transaminases to be decreased significantly in $60 \%$ cases with UDCA treatment. ${ }^{25}$

$30 \%$ of our patients had dyslipidemia. Dann AT et al also found in their study that total cholesterol levels raised more in OC significantly. ${ }^{26}$ This table shows $19 \%$ of the patients had deranged coagulation profile with increased PT or APTT level. Dang A et al $(29.78 \%)$ and Ray A et al (25\%) reported significant increased incidence of $\mathrm{PPH}$, as a result of malabsorption of vitamin $K$, due to steatorrhoea of cholestasis, leading to coagulation problem. ${ }^{23,24}$ Kenyon AP et al found a high incidence of $\mathrm{PPH}$ in OC patients who did not receive vitamin $\mathrm{K}$ compared to those who did (45\% vs $12 \%) .{ }^{3}$ Present study shows $10 \%$ incidence of PPH. Here, 19 patients had deranged coagulation profile and received vitamin $\mathrm{K}$, among them $4(21.05 \%)$ patients had PPH. Other 6 (7.4\%) patients who had PPH belongs to those 81 patients having normal coagulation parameters, hence not received vitamin K. PROM was found in $10 \%$ of cases in present study and $7 \%$ of patients had spontaneous preterm labour. Padmaja $\mathrm{M}$ et al reported a significant increase in incidence of PPROM (8.9\%) and PTL (44\%) in OC group. ${ }^{19}$

This study shows incidence of operative delivery (66\%), which comprises of Elective CS (32\%), Emergency CS $(30 \%)$ and Forceps delivery (4\%). It was significantly higher than that of VD $(34 \%)(\mathrm{p}<0.01)$. Kenyon AP et al found caesarean section rate $36.0 \%$. Rasheed $S$ et al reported spontaneous delivery rate of $80 \%$ with emergency LSCS rate of $16.7 \%$ and elective LSCS rate of $3.3 \% .^{3,21}$ It is often not clear whether this higher rate is as a result of active management or because of complications as a result of the disease. Caesarean section rate $(62 \%)$ was very high in present study, as like many UK hospitals, our tertiary private hospital also 
adopts a policy incorporating antenatal surveillance of some form with elective delivery by $37-38$ weeks. Hence, most of the patients $(62 \%)$ were with gestational age of delivery between $37-38$ weeks which was significantly higher $(\mathrm{p}<0.01)$ (mean 37.28 \pm 1.18 weeks).

Some studies, Roncaglia N et al and Fisk NM et al have reported good outcomes with a policy of induction of labour at 37 or $38 \mathrm{wk}$ gestation. ${ }^{27,28}$ Many clinicians in the UK have adopted this practice as the IUDs appear to cluster at later gestations. However there have been very few reports of the gestational week at which the IUD occurs, nor have there been any large prospective studies of whether drug treatment or early delivery prevents adverse fetal outcomes.

Fetal distress was found in $23 \%$ of the cases in present study, as well as abnormal CTG in $17 \%$ of cases. 41 patients had meconium stained liquor during delivery $(41.0 \%)$ which was significantly higher $(p<0.01)$. Alsulyman $\mathrm{OM}$ et al also found that risk of meconium passage was higher in the cholestasis group $(44.3 \%$ cases vs. $7.6 \%$ of control). ${ }^{29}$ It has been suggested that both fetal distress and increased gut motility by bile acids is the cause of raised incidence of MSL.

$22 \%$ of the babies has been suffering from prematurity (7\% preterm labour, $15 \%$ iatrogenetic due to fetal distress or abnormal CTG). This study shows that, mean birth weight of the babies were $2.80 \pm 0.36 \mathrm{~kg}$ (range 1.50-3.80 $\mathrm{kg}$ ). LBW (birth weight $<2.5 \mathrm{~kg}$ ) was found in $32 \%$ of the cases. Most of these babies had IUGR, and others had low birth weight due to preterm birth. Sosa S Y et al ${ }^{22}$ (2010) reported that neonates of OC mothers had an average weight of $2381 \pm 533 \mathrm{gm}$, while children of mothers in the control cohort had an average weight of $3118 \pm 470 \mathrm{gm}(\mathrm{p}>0.001)$. Williamson $\mathrm{C}$ et al observed $38 \%$ preterm delivery rate in cholestasis patients. ${ }^{30}$ IUFD or Still Born was found only in $2(2.0 \%)$ cases in the study, one at 33 weeks and another at 36 weeks. Alsulyman OM et al also found 2 of 79 patients having intrauterine fetal death in Obstetric Cholestasis group at 36 to 37 weeks of gestation. ${ }^{29}$ In $27 \%$ of the cases NICU admission were required in this study. According to Heinonen $\mathrm{S}$ et al intrahepatic cholestasis increases the need for intensive neonatal care in general population. ${ }^{20}$

In present study, in most of the case normal LFT $(98 \%)$ was found $(\mathrm{p}<0.01)$ after 6 weeks of delivery and symptomatically pruritus relieved. According to Rasheed $\mathrm{S}$ et al postnatal resolution of pruritus and liver function test occurred within 5-14 days with a mean of 8 $\operatorname{day} \pm 2.52 .^{21}$

\section{CONCLUSION}

In this study we came to appreciate that incidence of obstetric cholestasis is high in our hospital, as it is a tertiary care hospital, with high recurrence rate in multigravida patients. It is a disease of late pregnancy, mainly appears around 28-32 weeks gestation. There are several maternal morbidities detected as sleep disturbance, dyslipidemia, coagulation abnormality, PTL, PROM and PPH with increased rates of operative delivery. Maximum number of patients are delivered at 37 to 38 weeks, due to active maternal and fetal surveillance and early intervention to prevent sudden fetal death at late gestation. Neonatal morbidities are mainly due to fetal distress, prematurity, low birth weight and meconium staining of amniotic fluid. Fetal outcomes are improved with a variety of strategies of active management, although the most effective intervention has not currently been established. Ursodeoxycholic acid treatment is associated with marked improvement of symptoms and biochemical abnormalities. Almost all patients have postnatal resolution within 6 weeks of delivery.

However, Large therapeutic trials are required to establish which specific drug treatments or management strategies are effective at reducing the rates of adverse maternal and fetal outcomes. In this study the sample size is small, and the time period is limited-it therefore may not reflect the true magnitude of the problem; however, the 'take home message' is: early diagnosis and active maternal and fetal surveillance is of utmost importance to avoid adverse outcomes.

\section{ACKNOWLEDGMENTS}

Authors would like to thank Dr. Sukanta Misra Professor and HOD Gynecology and Obstetrics Department for his enumerable contribution, Dr. S. Mondal (statistician) for helping in statistical analysis and patients who take participation in this study.

Funding: No funding sources

Conflict of interest: None declared

Ethical approval: The study was approved by the Institutional Ethics Committee

\section{REFERENCES}

1. Lammert F, Marschall HU, Glantz A, Matern S. Intrahepatic cholestasis of pregnancy: molecular pathogenesis, diagnosis and management. J Hepatol. 2000;33:1012-21.

2. Beuers U, Pusl T. Intrahepatic cholestasis of pregnancy--a heterogeneous group of pregnancyrelated disorders. Hepatology. 2006;43:647-9.

3. Kenyon AP, Piercy CN, Girling J, Williamson C, Tribe RM, Shennan AH. Obstetric cholestasis, outcome with active management: a series of 70 cases. BJOG. 2002;109:282-8.

4. Ray A, Tata RJ, Balsara R. Cholestasis of pregnancy. J Obstet Gynecol India. 2005;55:247-50.

5. Greenes V, Williamson C. Intahepatic cholestasis of pregnancy. World J Gastroenterol. 2009;15(17):2049-66. 
6. Nichols AA. Cholestasis of Pregnancy: A Review of the Evidence. J Perinat Neonatal Nurs. 2005;19:21725.

7. Saleh MM, Abdo KR. Consensus on the management of obstetric cholestasis: National UK survey. BJOG. 2007;114:99-103.

8. Mays JK. The active management of intrahepatic cholestasis of pregnancy. Curr Opinion Obstet Gynecol. 2010;22:100-3.

9. Bacq Y, Sapey T, Brechot MC, Pierre F, Fignon A, Dubois F. Intrahepatic cholestasis of pregnancy: a French prospective study. Hepatology. 1997;26:358364.

10. Gonzalez MC, Reyes H, Arrese M, Figueroa D, Lorca B, Andresen M et al. Intrahepatic cholestasis of pregnancy in twin pregnancies. J Hepatol. 1989;9:84-90.

11. Glantz A, Marschall HU, Mattsson LA. Intrahepatic cholestasis of pregnancy: Relationships between bile acid levels and fetal complication rates. Hepatology. 2004;40:467-74.

12. Royal College of Obstetricians and Gynaecologists. Obstetric cholestasis. Green-Top Guideline No 43. 2006.

13. Lammert F, Marschall H, Matern S. Intrahepatic cholestasis of pregnancy. Curr Treat Options Gastroenterol. 2003;6:123-32.

14. Ahlfeld F. Reports and work from the obstetrics gynecological clinic in Giessen 1881-1882. Leipzig, Grunow FW;1883:148.

15. Abedin P, Weaver JB, Eggintin E. Intrahepatic cholestasis of pregnancy: prevalence and ethnic distribution. Ethnic Health. 1999;4:35-7.

16. Reyes H. Review: intrahepatic cholestasis. A puzzling disorder of pregnancy. J Gastroenterol Hepatol. 1997;12:211-6.

17. Germain AM, Carvajal JA, Glasinovic JC, Kato S, Williamson C. Intrahepatic cholestasis of pregnancy: an intriguing pregnancy-specific disorder. J Soc Gynecol Investig. 2002;9:10-14.

18. Gupta A, Kakkar T, Gupta Y, Hak J. Cholestasis of pregnancy. J Obstet Gynecol India. 2009 July/August;59(4):320-3.

19. Padmaja M, Pal B, Gupta JK, Ramamurthy S, Chaudhury M. A study of obstetric cholestasis. J Obstet Gynaecol India. 2010;60(3):225-31.
20. Heinonen S, Kirkinen P. Pregnancy outcome with intrahepatic cholestasis. Obstet Gynecol. 1999;94:189-93.

21. Rasheed S, Afghan S, Mazhar SB. Fetomaternal outcome in patients with obstetric cholestasis. Ann Pak Inst Med Sci. 2009;5(4):211-5.

22. Sosa SY, Valenzuela A, Pacheco J, Damián R. Intrahepatic Cholestasis of Pregnancy: Evaluation of Risk Factors and Predictive Factors. Internet J Gynecol Obstet. 2010;12(2):465-74.

23. Ray A, Tata RJ, Balsara R. Cholestasis of pregnancy. J Obstet Gynecol India. 2005;55:247-50.

24. Dang A, Agarwal N, Bathla S, Sharma N, Balani S. Prevalence of liver disease in pregnancy and its Outcome with emphasis on Obstetric Cholestasis : An Indian Scenario. J Obstet Gynecol India. 2010;60(6):413-8.

25. Deveer R, Engin-Ustun Y, Celen S, Eryilmaz OG, Tonguc E, Mollamahmutoglu L et al. Two-year experience of obstetric cholestasis: outcome and management. Clin Exp Obstet Gynecol. 2011;38(3):256-9.

26. Dann AT, Kenyon AP, Wierzbicki AS, Seed PT, Shennan AH, Tribe RM. Plasma lipid profiles of women with intrahepatic cholestasis of pregnancy. Obstet Gynecol. 2006;107:106-114.

27. Roncaglia N, Arreghini A, Locatelli A, Bellilni P, Andreotti C, Ghidini A. Obstetric cholestasis: outcome with active management. Eur J Obstet Gynecol Reprod Biol. 2002;100:167-70.

28. Fisk NM, Storey GNB. Fetal outcome in obstetric cholestasis. Br J Obstet Gynaecol. 1988;95:1137-43.

29. Alsulyman OM, Ouzounian JG, Ames Castro M, Goodwin TM. Intrahepatic cholestasis of pregnancy: perinatal outcome associated with expectant management. Am J Obstet Gynecol. 1996;175:95760.

30. Williamson C, Hems LM, Goulis DG, Walker I, Chambers J, Donaldson O et al. Clinical outcome in a series of cases of obstetric cholestasis identified via a patient support group. BJOG. 2004;111:676-81.

Cite this article as: Medda S, Sengupta S, Palo U. A study of the outcome of pregnancy complicated by obstetric cholestasis. Int J Reprod Contracept Obstet Gynecol 2018;7:996-1001. 